\title{
RELIABILITY OF THE GPS CARRIER-PHASE FIX SOLUTION UNDER HARSH CONDITION
}

\author{
Mohammad Ikmal Mohd Dzukhi ${ }^{1}$, Tajul Ariffin Musa ${ }^{1,2}$, Wan Anom Wan Aris ${ }^{1}$, Abdullah Hisam Omar ${ }^{1}$, Ivin Amri Musliman ${ }^{1}$ \\ ${ }^{1}$ Geomatic Innovation Research Group (UTM-GnG), Faculty of Built Environment and Surveying, Universiti Teknologi Malaysia \\ ${ }^{2}$ Centre of Tropical Geoengineering, Universiti Teknologi Malaysia
}

Commission 4, WG 7

KEY WORDS: Integer Ambiguity, Ambiguity validation, Ratio test, Fixed solution.

\begin{abstract}
Once the unknown integer ambiguity values are resolved, the GPS carrier phase observation will be transformed into a millimeterlevel precision measurement. However, GPS observation are prone to a variety of errors, making it a biased measurement. There are two components in identifying integer ambiguities: estimation and validation. The estimation procedure aims to determine the ambiguity's integer values, and the validation step checks whether the estimated integer value is acceptable. Even though the theory and procedures for ambiguity estimates are well known, the topic of ambiguity validation is still being researched. The dependability of computed coordinates will be reduced if a false fixed solution emerges from an incorrectly estimated ambiguity integer value. In this study, the reliability of the fixed solution obtained by using several base stations in GPS positioning was investigated, and the coordinates received from these bases were compared. In a conclusion, quality control measures such as employing several base stations will improve the carrier phase measurement's accuracy.
\end{abstract}

\section{INTRODUCTION}

Global Navigation Satellite System (GNSS) positioning, including Global Positioning System (GPS) is based on determining the distance between satellites and a receiver. Two types of distance measurements are used for this purpose; codebased pseudoranges and carrier-phase measurements. The observation of the code is based on a binary code modulated on the signal carrier. Using only code measurements, positioning can be done with several meters of precision. The difference between the phase of the receiver-produced carrier signal at reception time and the phase of the carrier signal generated in the satellite at transmission time is the carrier-phase measurement. It can be measured up to millimetre level.

The issue with carrier phase measurements is that each carrier phase has an ambiguous integer number of wavelengths. In addition, the carrier phase measurements are contaminated with many biases due to satellite, satellite to receiver, and receiver dependent errors. These errors complicate the process to estimate the integer number and thus it may lead to an incorrect integer value as well as wrong positioning results. Therefore, the most critical problem in precise GPS positioning is to address the integer ambiguities correctly.

Ambiguity resolution is described as the process of resolving unknown carrier phase cycle ambiguities to estimate the integer nature of the ambiguities, which is generally done in three (3) phases (Teunissen 2003). In the first phase, the integer nature of the ambiguity is ignored, and a normal least-square adjustment is performed to output float solutions, i.e., real number of ambiguity value, and their variance covariance matrix.

The real valued float solution of the ambiguity is further modified in the second phase to accommodate for the integer restriction. Consequently, a set of integer ambiguity values is obtained. The Integer Least Square (ILS) technique is one of the most often utilised integer estimators in practice. ILS has been demonstrated to maximise the likelihood of accurate integer estimate, making it the best approach for integer estimation (Atiz et al. 2021). Least-square AMBiguity Decorrelation Adjustment (LAMBDA) search algorithms are one of the most effective ILS approaches. The ambiguity validation procedure is carried out in the third phase after the integer ambiguity has been resolved, by checking the best and second best of the sets of integer candidates to ensure that the integer value is correct (Verhagen 2016). There are several ambiguity validations tests, including the integer aperture (IA) estimator, ratio test, F-ratio test, W-ratio, difference test, and projector test, with the ratio test presently being the most prevalent (Li and Wang 2012). However, because the statistical characteristics of ambiguity do not truly follow the normal distribution function, ambiguity validation remains an unresolved topic and further research is needed (Feng and Jokinen 2017; Verhagen 2016, 2004).

\section{AMBIGUITY VALIDATION}

Ambiguity validation is a process of applying an acceptance test to validate the reliability of an estimated integer ambiguity. Three (3) hypotheses are identified in validating the float and fixed solution; 1) assuming that all unknown parameter are real-valued, 2) integer constraint is considered by assuming that the correct integer values of the ambiguity are known, and 3) to not put any restriction on the integer value (Teunissen and Kleusberg 1998). Several validity tests have been proposed based on these hypotheses, and they are continually improving.

\section{$2.1 F$-ratio test}

The first approach proposed for ambiguity validation is the $F$ ratio test by Frei and Beutler (1990) which is the ratio between $\Omega_{1}$ and $\Omega_{2}$.

$$
F=\frac{\Omega_{1}}{\Omega_{2}}
$$


Where $\Omega_{1}=$ minimum quadratic form of residual in the ambiguity fixed solution

$\Omega_{2}=$ second minimum quadratic form of residual in the ambiguity fixed solution

Normally, an $F$-distribution is considered as the test coefficient for $F$-ratio, with degrees of freedom for $\Omega_{1}$ and $\Omega_{2}$ respectively. Then, by defining a significance level, a threshold value c could be obtained. The numerator and denominator are not distinct, according to Teunissen (1998); Wang, Stewart, and Tsakiri (1998) and the $F$-ratio does not obey an $F$-distribution. Even if such analytical values $(1.5,2$ and 3$)$ behave satisfactorily, there are often significant differences between the obtained results and their true values, indicating that the $F$-ratio is not accurate (Leick 2015)

\subsection{W-ratio test}

Another classification technique suggested by Wang, Stewart, and Tsakiri (1998) is to compare two likelihoods of two integer candidates or to artificially nest the two compared models using a nesting parameter. $W$-ratio is defined as follows

$$
\begin{aligned}
W & =\frac{d}{\sqrt{\operatorname{Var}(d)}} \\
d & =\Omega_{1}-\Omega_{2}
\end{aligned}
$$

If $W_{1}$ and $W_{2}$ are the two ratios that correspond to a priori and a posteriori deviation, they should have a truncated regular normal distribution and a truncated student t-distribution, respectively, from which the critical values can be easily derived (Li and Wang 2012). The $W$-ratios will have a rigorous confidence standard for the validation test under the premise that the set ambiguities are deterministic quantities.

\section{$2.3 R$-ratio test}

The R-ratio test was proposed by Euler and Schaffrin (1991) and is one of the famous techniques used for ambiguity validation test. This evaluation generates a value by dividing the best and second-best solutions. User must set a threshold value, and if the results surpass the threshold value, the ambiguity sets are discarded and re-estimated. The ratio test read as:

$$
R=\frac{R_{1}}{R_{2}} \geq c
$$

The threshold value is normally set by the user and according to research, there is still no solid number to be set as the threshold value. Several suggested threshold values were proposed such as 1.5 (Han and Rizos 1996), 2 (Wei and Schwarz 1995), 3 (Leick, Rapoport, and Tatarnikov 2015) and 2.5 (Parkins 2011). However, based on many real-case experiments performed by experts, it is recommended that the threshold value is set at 3.0. In fact, if the ratio is greater than 3 , the integer ambiguity is most likely correct but not necessary; if the ratio is less than 3 , the integer ambiguity is most likely incorrect but not necessary.

Due to the difficulty in determining the threshold value, these ratio tests are still an issue (Verhagen and Teunissen 2006). The disadvantage of employing a set c-value ratio test is that, depending on the quality of the model, either the failure rate or the false alarm rate will become too high. Controlling the failure rate is especially essential since erroneous integer estimates might have a substantial impact on the baseline solution. As a result, a ratio test with a fixed failure rate was created (Teunissen and Verhagen 2009; Verhagen and Teunissen 2006). This fixed- failure ratio test (FF-RT) uses a model-driven c-value to verify that the failure rate does not exceed a user-defined threshold. As the ambiguity validation race heats up, Li et al, (2016) propose the Integrity Monitoring-based Ratio Test (IM-RT) as an alternative method for validating the ambiguity's dependability, since neither the ratio test nor the FF-RT would properly evaluate the efficiency of ambiguity resolution (AR). As evidenced by the ideal balancing performance between false alarm and missing detection errors, simulation findings demonstrate that IM-RT has better ambiguity validation efficiency than FF-RT and ratio test.

\subsection{Difference test}

Difference test is another validation approach to analyse the difference between the best $\left(R_{1}\right)$ and second-best $\left(R_{2}\right)$ sets of ambiguity integer value. A global model evaluation is performed first. The uncertainty could then be checked by comparing the difference test to its critical value, as below

$$
R_{1}-R_{2} \geq c
$$

where $\mathrm{c}$ is a user-defined tolerance level and is a non-negative scalar. If the float solution is much closer to the best solution than the second-best solution, this measure accepts the best solution. Tiberius and De Jonge (1995) and Han (1997) proposed threshold values of 15 and 12 . However, since the distribution of the difference test is uncertain, the estimation of the critical value, which is also observational, is contentious. A looser collection of critical principles improves results, but it also raises the number of applicants that are mistakenly admitted (Type 1 errors).

\section{QUALITY CONTROL IN GPS MEASUREMENT}

Since the ambiguity validation test is still an unresolved issue due to its ambiguity statistical properties, there is the need of checking the method to validate the output coordinate. The positioning accuracy of an output coordinate from an incorrect fixed solution might vary by tens of centimetres or several metres. As a result, to preserve the carrier phase measurement's dependability, a quality check in GPS measurement is required. This quality control will be based on a practical method, in which the measurement's output will be examined.

There are several ideas on quality control in GPS positioning which includes a check for ambiguity on each initialization and repeat profiling. Obtaining the coordinates of a point with a different initialization is one way to achieve it. After that, the coordinates acquired from both initializations were examined. If there is a significant disparity between the two coordinates, one of the solutions obtained is incorrectly fixed. As a result, another initialization is needed to ensure the location coordinate is correct. The Department of Survey and Mapping Malaysia used the same approach in establishing Cadastral Reference Marks in the circular (PKPUP bil.6 2009).

Furthermore, additional Continuous Operating Reference Station (CORS) or base stations can be used to do independent checks. Using two (2) or more base stations will result in a robust network and redundant observational measurements. In an example where many base stations are utilised in Real-Time Kinematic (RTK) GPS techniques, the outcome obtained includes a large number of resulting coordinates with regard to the base stations. By comparing the coordinates acquired from all the bases, an independent verification can be performed. If it's used in network processing, the more CORS that are used, the stronger the network becomes. According to study done by Lotfy, Abdelfatah, and Hosny (2019), independent checking using multiple base 
stations has been successful in increasing the coordinate's trustworthiness. Based on their findings, when three (3) base stations are used, the mean and standard deviation of coordinates acquired are substantially lower than when only one (1) base station is used.

\section{TEST RESULT AND ANALYSIS}

A test was done in August 2019 at Universiti Teknologi Malaysia in Skudai, Johor, to demonstrate the fix solution's reliability in harsh conditions. For 15 minutes, GPS rapid static observations are carried out at 30 specified points under varying observing conditions (Figure 1). To resolve the baseline solution, two (2) base stations were used, with one (1) of the base stations acting as a checking base station. The baseline solution was resolved using standard GPS processing software to establish the coordinates of the designated point. Due to developer confidentiality, the ambiguity resolution technique utilised by this software is unknown, however we believe they used the best approach among the best for the benefit of the users. As a result, all point solutions are classified into three (3) categories: true fix, questionable fix, and wrong fix solution. These classifications are based on the difference in coordinates found when comparing result from two (2) bases.

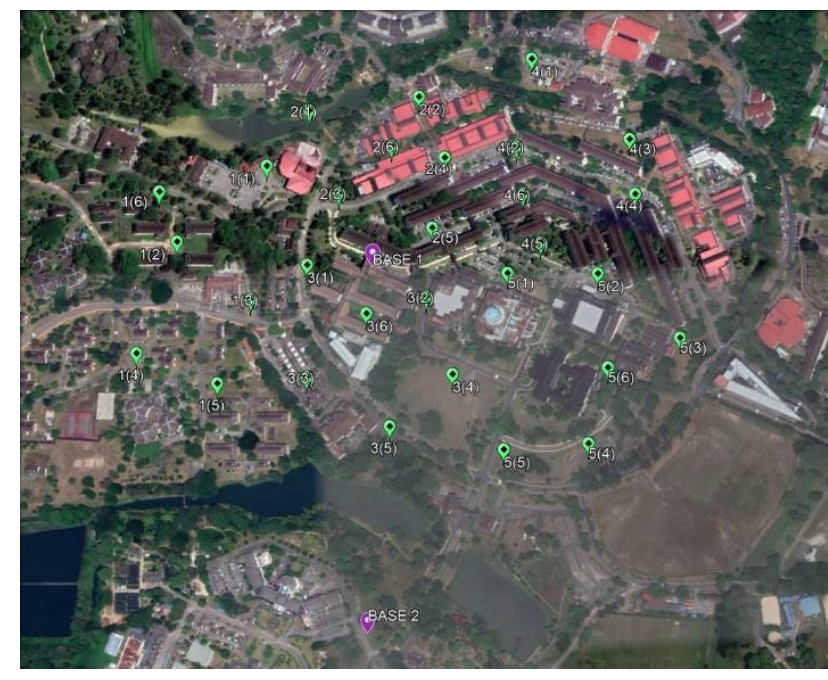

Figure 1. GPS observation point distribution.

\begin{tabular}{|c|c|c|c|c|c|c|c|c|}
\hline \multirow{2}{*}{ Point ID } & \multicolumn{3}{|c|}{ BASE 1} & \multicolumn{3}{|c|}{ BASE 2} & \multicolumn{2}{|c|}{ Coordinate Difference $(\mathrm{m})$} \\
\hline & Solution Type & H. Precision $(95 \%)(\mathrm{m})$ & V. Precision (95\%)(m) & Solution Type & H. Precision (95\%)(m) & V. Precision (95\%)(m) & Northing & Easting \\
\hline $1(1)$ & Fixed & 0.002 & 0.002 & Fixed & 0.002 & 0.002 & 0.014 & 0.005 \\
\hline $1(2)$ & Fixed & 0.008 & 0.021 & Fixed & 0.006 & 0.015 & 0.014 & 0.003 \\
\hline $1(2)$ & Fixed & 0.006 & 0.017 & Fixed & 0.007 & 0.020 & 0.014 & 0.004 \\
\hline $1(4)$ & Fixed & 0.002 & 0.003 & Fixed & 0.002 & 0.003 & 0.014 & 0.002 \\
\hline $1(5)$ & Fixed & 0.002 & 0.003 & Fixed & 0.002 & 0.002 & 0.015 & 0.003 \\
\hline $1(6)$ & Fixed & 0.005 & 0.014 & Fixed & 0.004 & 0.010 & 0.015 & 0.001 \\
\hline $2(1)$ & Fixed & 0.012 & 0.035 & Fixed & 0.013 & 0.038 & 0.009 & -0.002 \\
\hline $2(2)$ & Fixed & 0.003 & 0.007 & Fixed & 0.003 & 0.005 & 0.015 & 0.002 \\
\hline $2(3)$ & Fixed & 0.002 & 0.002 & Fixed & 0.002 & 0.003 & 0.016 & 0.002 \\
\hline $2(4)$ & Fixed & 0.002 & 0.006 & Fixed & 0.002 & 0.005 & 0.014 & 0.002 \\
\hline $2(5)$ & Fixed & 0.002 & 0.006 & Fixed & 0.003 & 0.007 & 0.013 & 0.004 \\
\hline 2(6) & Fixed & 0.013 & 0.025 & Fixed & 0.009 & 0.018 & 0.015 & 0.001 \\
\hline $3(1)$ & Fixed & 0.003 & 0.004 & Fixed & 0.002 & 0.003 & 0.016 & 0.003 \\
\hline $3(2)$ & Fixed & 0.005 & 0.015 & Fixed & 0.003 & 0.008 & 0.019 & -0.001 \\
\hline $3(3)$ & Fixed & 0.002 & 0.004 & Fixed & 0.002 & 0.003 & 0.015 & 0.002 \\
\hline $3(4)$ & Fixed & 0.002 & 0.002 & Fixed & 0.002 & 0.002 & 0.013 & 0.004 \\
\hline $3(5)$ & Fixed & 0.003 & 0.008 & Fixed & 0.002 & 0.006 & 0.018 & 0.004 \\
\hline $3(6)$ & Float & 4.299 & 0.646 & Fixed & 0.001 & 0.001 & -2.209 & -2.524 \\
\hline $4(1)$ & Fixed & 0.005 & 0.013 & Fixed & 0.003 & 0.008 & 0.010 & -0.005 \\
\hline $4(2)$ & Fixed & 0.003 & 0.007 & Fixed & 0.002 & 0.006 & 0.013 & 0.004 \\
\hline $4(3)$ & Fixed & 0.002 & 0.004 & Fixed & 0.002 & 0.003 & 0.016 & -0.001 \\
\hline $4(4)$ & Fixed & 0.004 & 0.009 & Fixed & 0.003 & 0.006 & 0.015 & 0.006 \\
\hline $4(5)$ & Fixed & 0.003 & 0.004 & Fixed & 0.003 & 0.005 & 0.013 & 0.002 \\
\hline $4(6)$ & Fixed & 0.006 & 0.005 & Fixed & 0.001 & 0.001 & -0.576 & -0.514 \\
\hline $5(1)$ & Fixed & 0.003 & 0.007 & Fixed & 0.004 & 0.011 & 0.012 & 0.001 \\
\hline $5(2)$ & Fixed & 0.004 & 0.013 & Fixed & 0.004 & 0.015 & 0.016 & 0.006 \\
\hline $5(3)$ & Fixed & 0.003 & 0.008 & Fixed & 0.003 & 0.006 & 0.012 & -0.003 \\
\hline $5(4)$ & Fixed & 0.003 & 0.003 & Fixed & 0.002 & 0.003 & 0.015 & 0.004 \\
\hline $5(5)$ & Fixed & 0.002 & 0.005 & Fixed & 0.002 & 0.005 & 0.015 & -0.001 \\
\hline $5(6)$ & Fixed & 0.006 & 0.010 & Fixed & 0.004 & 0.007 & 0.019 & -0.002 \\
\hline
\end{tabular}

Table 1. Processing result of 30 GPS observation point together with coordinate difference.

\subsection{True fix solution}

During the observation, only 28 out of 30 points were able to reach a fixed solution (Table 1). The fixed solution obtained from these 28 sites are evident since majority of them were observed under a clear sky view. There were also some points that were severely obstructed by nearby buildings and trees, but the algorithm in the software was able to resolve the ambiguity to an integer number. Because of the slight discrepancies between the two bases, the coordinates obtained from both are sufficiently trustworthy.

\subsection{Questionable fix solution}

Table 1 shows only one (1) point, point ID 4(6) and was identified as a dubious fixed solution. The software's algorithm was able to resolve the integer ambiguity from both BASE 1 and 
BASE 2 baselines although it had been observed under harsh condition (Figure 2). However, there is a discrepancy of -0.576 $\mathrm{m}$ in the North component and $-0.514 \mathrm{~m}$ in the East component between the two coordinates. Both BASE 1 and BASE 2 solutions have a precision of less than $0.006 \mathrm{~m}$ on horizontal and vertical components. As a result, neither the BASE 1 nor BASE 2 coordinates will be chosen as a genuine fixed solution. In order to confirm the resulting coordinates from these bases, it is proposed that a third base station be established.

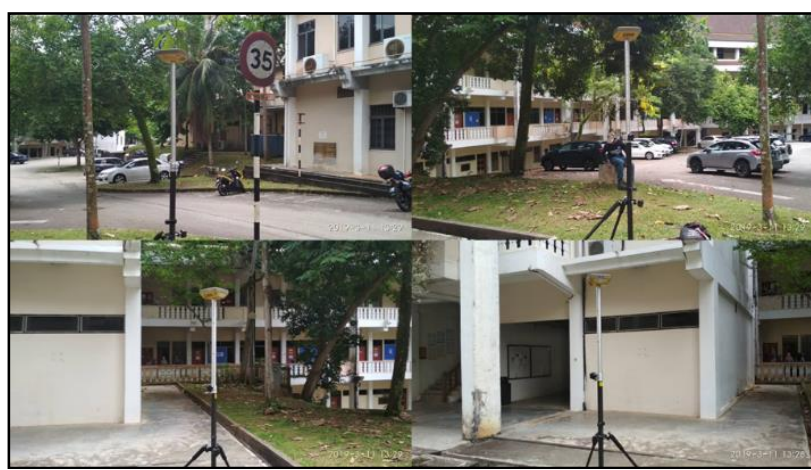

Figure 2. GPS observation at point 4(6)

\subsection{Wrong fix solution}

Only one (1) point, point ID 3(6), was recognized as an incorrect fixed solution in Table 1. However, this instance differs from the others in that a fixed solution could only be obtained through observation with regard to BASE 2, whereas BASE 1 yielded a float solution. Despite the significant coordinate difference of around 2 meters, the software recorded the solution as a fixed solution. Therefore, fixed solutions in respect of BASE 2 are considered false fixed solutions based on these findings. This is because the observation location has a relatively restricted sky view. It was surrounded by high-rise buildings and tall trees (Figure 3), and it could only receive signals from high-altitude satellites, although some of them had multiple cycle slips.

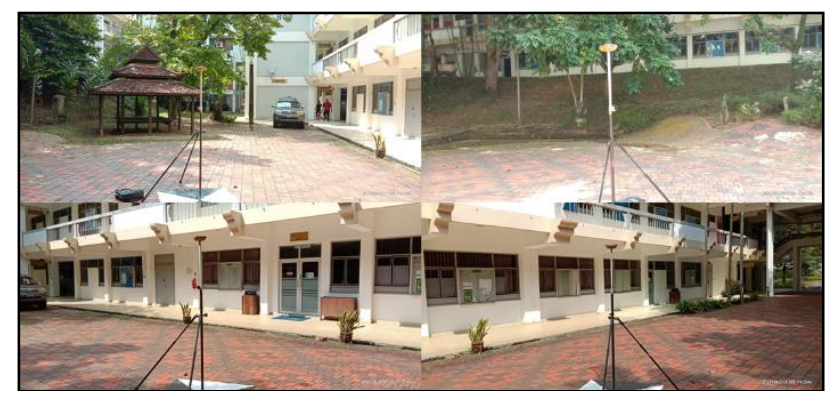

Figure 3. GPS observation at point 3(6)

\section{SUMMARY}

The race to improve the ambiguity validation test is still on-going because all present tests have statistical problems, but they still fulfil the user's needs. Although the points were observed under difficult conditions, the advanced and newest processing technique incorporated in the processing programme was capable of resolving the ambiguity to its integer value. Yet, due to an incorrectly estimated integer value, the fixed solution obtained may at times be false. As a result, a practical verification technique involving the use of a second base station to confirm the obtained coordinates is required. An additional base station will aid in determining the incorrect fixed solution and the reoccupation of that station. However, because this method has only been tested on post-processing techniques, more research is needed to extend this validation method to real-time kinematic techniques.

\section{ACKNOWLEDGEMENTS}

The author would like to thank Geomatic Innovation Research Group (UTM-GnG) in providing the GNSS data for this study. The author also would like to channel an appreciation towards Universiti Teknologi Malaysia and Research University Grant (RUG) vot no. R.J13000.7352.4J341 for funding this research study.

\section{REFERENCES}

Atiz, Omer Faruk, Sermet Ogutcu, Salih Alcay, Pan Li, and Ilkay Bugdayci. 2021. 'Performance investigation of LAMBDA and bootstrapping methods for PPP narrow-lane ambiguity resolution', Geo-spatial Information Science: 1-10.

Euler, H-J, and B Schaffrin. 1991. 'On a measure for the discernibility between different ambiguity solutions in the statickinematic GPS-mode.' in, Kinematic systems in geodesy, surveying, and remote sensing (Springer).

Feng, Shaojun, and Altti Jokinen. 2017. 'Integer ambiguity validation in high accuracy GNSS positioning', GPS solutions, 21: 79-87.

Frei, E., and G. Beutler. 1990. 'Rapid static positioning based on the fast ambiguity resolution approach FARA: theory and first results', Manuscripta geodaetica, 15: 325-56.

Han, Shaowei. 1997. 'Quality-control issues relating to instantaneous ambiguity resolution for real-time GPS kinematic positioning', Journal of geodesy, 71: 351-61.

Han, Shaowei, and Chris Rizos. 1996. 'Validation and rejection criteria for integer least-squares estimation', Survey Review, 33: 375-82.

Leick, Alfred, Lev Rapoport, and Dmitry Tatarnikov. 2015. GPS satellite surveying (John Wiley \& Sons).

Li, Liang, Zishen Li, Hong Yuan, Liang Wang, and Yanqing Hou. 2016. 'Integrity monitoring-based ratio test for GNSS integer ambiguity validation', GPS solutions, 20: 573-85.

Li, T, and J Wang. 2012. 'Some remarks on GNSS integer ambiguity validation methods', Survey Review, 44: 230-38.

Lotfy, A, MA Abdelfatah, and H Hosny. 2019. 'Quality Control of Kinematic GNSS Networks: a case study of the regional network at Sharqia Governorate in Egypt'.

Parkins, Alex. 2011. 'Increasing GNSS RTK availability with a new single-epoch batch partial ambiguity resolution algorithm', GPS solutions, 15: 391-402.

Teunissen, Peter JG. 1998. 'Success probability of integer GPS ambiguity rounding and bootstrapping', Journal of geodesy, 72: 606-12. 
Teunissen, Peter JG, and Alfred Kleusberg. 1998. 'GPS observation equations and positioning concepts.' in, GPS for Geodesy (Springer).

Teunissen, PJG. 2003. 'Theory of carrier phase ambiguity resolution', Wuhan University Journal of Natural Sciences, 8: 471-84.

Teunissen, PJG, and S Verhagen. 2009. 'GNSS carrier phase ambiguity resolution: challenges and open problems.' in, Observing our changing Earth (Springer).

Tiberius, CCJM, and PJ De Jonge. 1995. 'Fast positioning using the LAMBDA method', Proceedings DSNS-95, paper, 30.

Verhagen, Sandra. 2004. 'Integer ambiguity validation: an open problem?', GPS solutions, 8: 36-43.

2016. 'GNSS Ambiguity Resolution and Validation.' in, Encyclopedia of Geodesy (Springer).

Verhagen, Sandra, and Peter JG Teunissen. 2006. 'New global navigation satellite system ambiguity resolution method compared to existing approaches', Journal of Guidance, Control, and Dynamics, 29: 981-91.

Wang, J, MP Stewart, and M Tsakiri. 1998. 'A discrimination test procedure for ambiguity resolution on-the-fly', Journal of geodesy, 72: 644-53.

Wei, Ming, and Klaus-Peter Schwarz. 1995. "Fast ambiguity resolution using an integer nonlinear programming method." In Proceedings of the 8th International Technical Meeting of the Satellite Division of the Institute of Navigation (ION GPS 1995), 1101-10. 